\title{
Role of Molecular Chaos in Granular Fluctuating Hydrodynamics
}

\author{
G. Costantini and A. Puglisi *
}

CNR-ISC and Dipartimento di Fisica, Università Sapienza - p.le A. Moro 2, 00185, Roma, Italy

\begin{abstract}
We perform a numerical study of the fluctuations of the rescaled hydrodynamic transverse velocity field during the cooling state of a homogeneous granular gas. We are interested in the role of Molecular Chaos for the amplitude of the hydrodynamic noise and its relaxation in time. For this purpose we compare the results of Molecular Dynamics (MD, deterministic dynamics) with those from Direct Simulation Monte Carlo (DSMC, random process), where Molecular Chaos can be directly controlled. It is seen that the large time decay of the fluctuation's autocorrelation is always dictated by the viscosity coefficient predicted by granular hydrodynamics, independently of the numerical scheme (MD or DSMC). On the other side, the noise amplitude in Molecular Dynamics, which is known to violate the equilibrium Fluctuation-Dissipation relation, is not always accurately reproduced in a DSMC scheme. The agreement between the two models improves if the probability of recollision (controlling Molecular Chaos) is reduced by increasing the number of virtual particles per cells in the DSMC. This result suggests that DSMC is not necessarily more efficient than MD, if the real number of particles is small $\left(\sim 10^{3} \pm 10^{4}\right)$ and if one is interested in accurately reproduce fluctuations. An open question remains about the small-times behavior of the autocorrelation function in the DSMC, which in MD and in kinetic theory predictions is not a straight exponential.
\end{abstract}

Key words: granular gas, fluctuations, hydrodynamics, numerical simulations AMS subject classification: 76P05, 82-08, 82D30

\footnotetext{
*Corresponding author. E-mail: Andrea.Puglisi@roma1.infn.it
} 


\section{Introduction}

A relevant question for the general problem of non-equilibrium statistical mechanics is: may two non-equilibrium statistical processes be considered to be in the same "class of universality"? This question is usually more complicate than the analogous problem at equilibrium, because less symmetries are available, the most important being the invariance under time-reversal. Models of granular gases $[30,29]$ lend themselves to be analyzed under this aspect: they are usually far from equilibrium and exhibit peculiar properties, such as: breakdown of energy equipartition [16, 25, 28], spontaneous symmetry breaking (vortices and clustering) [35, 18], "Maxwell demon" effects [15], "ratcheting" effects [12], and lot more. At the same time granular experiments usually concern small samples, or the order of $10^{3} \pm 10^{4}$ grains, and therefore extensive observables are affected by large (easily detectable) fluctuations [18]. Finding "universal" properties in the statistical description of those fluctuations is, therefore, of great interest. Recently a kinetic theory has been proposed for the fluctuations of "slow" (hydrodynamics) fields in a uniform, dilute and unforced granular gas made of inelastic hard disks or spheres [5]. The system - initialized in a generically thermal state - cools down; during the cooling the fields can be properly rescaled to fit a steady description. The theory predicts a form, for the fluctuations of the fields, which is different from the classical Landau theory for fluctuating hydrodynamics [21], which is based on Onsager reciprocal relations or equivalently the Kubo Fluctuation-Dissipation relation (FDR) [20, 26]. The fluctuations obey, instead, a generalized Langevin equation where the noise has a variance which does not satisfy the FDR [14, 3], and, in addition, is slightly colored. Note that the failure of FDR has also been observed in driven granular gases, but only at large packing fractions [32, 36, 34], while it is usually satisfied in the dilute case [31, 1,33]. The theory in [5] makes use of the projection operator formalism and descends from a series of hypothesis, mainly the validity of Molecular Chaos and the Liouville equation for free particles undergoing hard core binary instantaneous collisions. It well reproduces the results of Molecular Dynamics simulations. A different model, often used to describe a homogeneous and dilute granular gas, is the one known as non-homogeneous Direct Simulation Monte Carlo for inelastic hard disks (or spheres) [2]: even if this is usually considered a numerical scheme good to reproduce hard disks MD in the dilute limit, from the point of view of fluctuations it is clearly a different model. Indeed, in an MD simulation the noise is simply generated by the dynamics (with different initial conditions), while the DSMC introduces an external source of noise to randomize collisions. Observables relying on the one-particle distribution function (which satisfies the same evolution equation, i.e. the inelastic Boltzmann equation) are expected to take the same values in MD and DSMC. Anyway fluctuations of hydrodynamic fields are related to two-particles distribution functions: at this level of the BBGKY hierarchy, the relation between DSMC and MD is still to be assessed. For this reason we have performed a detailed study of hydrodynamics noise comparing DSMC and MD. Our study has been in part published in [11]: here we add results concerning the role of the number of cells $\left(m_{c}\right)$ and of the virtual particles per cell $\left(\tilde{N}_{c}\right)$, which change the accuracy of the DSMC model in reproducing the Molecular Chaos. It appears that reducing the probability of recollision by tuning these parameters is sufficient to improve the agreement between DSMC and MD. Previously, DSMC has been used to measure hydrodynamic fluctuations in the elastic case [17, 24]. More recently, the DSMC approach has 
also been applied to fluctuations of the global energy fluctuations (a homogeneous, not spatially dependent quantity) in inelastic systems [37, 10], obtaining a good agreement with the amplitude of fluctuations measured in MD simulations and explained in terms of projection operators applied to the deterministic dynamics [9]. A similar conclusion was available for that study: stochastic "Monte Carlo" treatment of collisions is sufficient to reproduce MD noise properties. A successive study [13] also showed how certain quantities (cumulants of fluctuations in the stationary state) may be obtained without resorting to projection techniques. The novelty of the present case is in the local nature of the observable: fluctuations are caused by evolution of the spatial coordinates and not only of the velocity degrees of freedom. The organization of the paper is the following: we first describe the model in Section 2., explaining the stationary HCS representation and the two numerical schemes in use; then, in Section 3., we discuss the role of spatial resolution and how it affects Molecular Chaos; then the hydrodynamic noise is discussed in in Section 4. and finally conclusions are drawn.

\section{The homogeneous cooling and its stationary representation}

We treat, by Direct Simulation Monte-Carlo (DSMC) and Event Driven Molecular Dynamics (MD) the evolution of a dilute system of $N$ smooth inelastic hard disks of mass $m=1$ and diameter $\sigma$. The instantaneous inelastic collisions between the disks $i$ and $j$ change the particles velocities following the relations

$$
\begin{aligned}
\mathbf{v}_{i}^{\prime} & =\mathbf{v}_{i}-\frac{1+\alpha}{2}\left(\hat{\sigma} \cdot \mathbf{v}_{i j}\right) \hat{\sigma} \\
\mathbf{v}_{j}^{\prime} & =\mathbf{v}_{j}+\frac{1+\alpha}{2}\left(\hat{\sigma} \cdot \mathbf{v}_{i j}\right) \hat{\sigma}
\end{aligned}
$$

where $\mathbf{v}^{\prime}$ corresponds to post-collisional velocity, $\alpha$ is the coefficient of restitution and $\mathbf{v}_{i j}=\mathbf{v}_{i}-\mathbf{v}_{j}$ is the relative velocity. The term $\hat{\sigma}$ is the unit vector joining the particles at contact, in the MD, and a random unit vector (see details below) in the DSMC. The size of the system is $L \times L$ and the boundary conditions are periodic. The main hypothesis used here is that the system, starting in a uniform equilibrium state with granular temperature $T_{g}(0)=T_{0}$, evolves to the Homogeneous Cooling State (HCS), which is characterized by a single time-scale measured by the temperature $T_{g}(t)$ : any other quantity depends on time only through $T_{g}(t)$. Apparently, as observed in many previous studies [8], in a homogeneous setting, Molecular Chaos is sufficient to guarantee this hypothesis. The HCS is unstable against spatial fluctuations: this instability appears at scales larger than a critical length $L_{c}$ which depends on $\alpha$ and on the mean free path, therefore it can be avoided by taking the linear size of the system $L<L_{c}$ [19]. It is possible to analyze the effects of spatial fluctuations by deriving mesoscopic equations through a linearization around the HCS [35]. The resulting equations are generally coupled, but in the Fourier representation the transverse velocity field results decoupled from the other modes. We are interested, in particular, in the fluctuations of its largest mode, i.e. of the smallest wave-number $\left|\mathbf{k}_{m}\right|=2 \pi / L$. We choose the wave vector $\mathbf{k}_{m}$ 
parallel to the axis $\hat{x}$ and the quantity above results to be

$$
U_{\perp}(t)=\sum_{j=1}^{N} v_{y, j}(t) \exp \left(i \frac{2 \pi x_{j}(t)}{L}\right)
$$

where $v_{y, j}(t)$ is the component of the velocity of the particle $j$ in the direction $\hat{y}$ and $x_{j}(t)$ is its coordinate along the $\hat{x}$ axis. According to the Landau-Lifshitz Equilibrium Fluctuating Hydrodynamics $(\mathrm{EFH})$, based on Einstein fluctuation formula, one can make the hypothesis that the fluctuations of $U_{\perp}(t)$ obey a linear Langevin equation:

$$
\partial_{t} U_{\perp}(t)=-\nu(t) k_{m}^{2} U_{\perp}(t)+\sqrt{v_{t h}^{2}(t) N c \nu(t) k_{m}^{2}} \xi(t)
$$

where $\nu(t)$ is the kinematic viscosity which, in the HCS, is proportional to $\sqrt{T_{g}}$ (see [4] for definitions), $v_{t h}(t) \equiv \sqrt{2 T_{g} / m}$ and $c$ is expected to be $1[21,35,3]$. The last term in the Eq. (2.3) describes an internal noise due to the effect of the rapid (microscopic) degrees of freedom of the system which have been projected out of the description. This complex noise is assumed, in EFH, to be white, Gaussian and with correlations given by

$$
\begin{aligned}
\left\langle\xi(t) \xi^{*}\left(t^{\prime}\right)\right\rangle & =\delta\left(t-t^{\prime}\right) \\
\left\langle\xi(t) \xi\left(t^{\prime}\right)\right\rangle & =0
\end{aligned}
$$

with $\xi^{*}(t)$ the complex conjugate of $\xi(t)$. In granular systems, deviations from the white noise assumption are expected [5], but in the dilute case they are in general quite small (this is different in stationary dense systems, e.g. [32, 36, 34]). The most evident consequence of inelasticity is, instead, the variation of the dimensionless coefficient $c$ in Eq. (2.3), which is equal to 1 only at equilibrium $(\alpha=1)$; its departure from $c=1$ represents the fact that the fluctuations of $U_{\perp}(t)$ do not satisfy the FDR and that the EFH fails. Since the system is cooling, the typical velocity of the particles becomes smaller and smaller, leading to increasing rounding errors and other numerical problems. These problems can be avoided with the use of a procedure which, in the HCS, maps the dynamics to a steady state by means of a time-rescaling $[8,23]$. In the HCS, the granular temperature obeys the following equation:

$$
\partial_{t} T_{g}(t)+\zeta_{H}(t) T_{g}(t)=0
$$

where $\zeta_{H}$ is the cooling rate that results proportional to $\sqrt{T_{g}(t)}$.

The stationary representation of the HCS [8] consists in introducing a new time scale $\tau$ defined by

$$
\omega_{0} \tau=\ln \frac{t}{t_{0}}
$$

with $\omega_{0}$ ant $t_{0}$ arbitrary constants, implying the definition of rescaled velocities $\tilde{\mathbf{v}}(\tau)=\mathbf{v}(t) \omega_{0} t$. It is easy to see [8] that observing the system on this new time scale is equivalent to apply a positive continuous drag to all particles $\partial_{\tau} \tilde{\mathbf{v}}(\tau)=\omega_{0} \tilde{\mathbf{v}}(\tau)$. This naturally leads to define also the rescaled 
analogous of $U_{\perp}(t): W_{\perp}(\tau)=U_{\perp}(t) \omega_{0} t$.

We tune $\omega_{0}$ in order to have $T_{g}(\tau \rightarrow \infty)=T_{0}$ (so that the length of transients is reduced), a result which is obtained by taking $\omega_{0}=\zeta_{H}(0) / 2$. In the steady state, we simplify the notation using $v_{t h}=v_{t h}(0)$. With these choices, the Langevin equation (2.3) is mapped onto a new equation

$$
\partial_{\tau} W_{\perp}(\tau)=-\frac{W_{\perp}(\tau)}{\tau_{0}}+\sqrt{N v_{t h}^{3} \frac{c \tilde{\nu} \tilde{k}_{m}^{2}}{\lambda_{0}}} \xi(\tau),
$$

where $\tau_{0}^{-1}=\frac{v_{t h}}{\lambda_{0}} \tilde{\nu} \tilde{k}_{m}^{2}-\omega_{0}, \lambda_{0}=L^{2} /(N \sigma) \equiv 1 /(n \sigma)$ is proportional to the mean free path and $\tilde{\nu}=\nu(t) /\left[\lambda_{0} v_{t h}(t)\right]$ and $\tilde{k}_{m}=k_{m} \lambda_{0}$ are dimensionless rescaled viscosity and wave number, respectively. If $\tau_{0}>0$, which is equivalent to the condition of stability of shear modes in the HCS, the above equation leads to a dimensionless autocorrelation function given by

$$
C_{\perp}(\tau) \equiv \frac{\left\langle W_{\perp}(0) W_{\perp}^{*}(\tau)\right\rangle}{v_{t h}^{2}}=\frac{c N}{2}\left(1+\omega_{0} \tau_{0}\right) e^{-\tau / \tau_{0}}
$$

with $\tau_{0}$ the characteristic time of decay.

Based on Eq. (2.9), we can obtain the dimensionless kinematic viscosity $\tilde{\nu}$ and the "FDR violation" coefficient $c$ from a measure of $C_{\perp}(\tau)$, fitting its main parameters $C_{\perp}(0)$ and $\tau_{0}$ :

$$
\begin{aligned}
\tilde{\nu} & =\frac{\lambda_{0}}{v_{t h} \tilde{k}_{m}^{2}}\left(\frac{1}{\tau_{0}}+\omega_{0}\right) \\
c & =\frac{2 C_{\perp}(0)}{N\left(\tau_{0} \omega_{0}+1\right)}
\end{aligned}
$$

i.e. $\tilde{\nu}$ and $c$ are obtained measuring the amplitude $C_{\perp}(0)$ and the decay time $\tau_{0}$. In order to apply the correct thermostat $\dot{\tilde{\mathbf{v}}}=\omega_{0} \tilde{\mathbf{v}}$, and to obtain the kinematic viscosity $\tilde{\nu}$ from Eq.(2.9), it is necessary to know the value of $\omega_{0}=\zeta_{H}(0) / 2$. This is computed, in the homogeneous case, giving the theoretical expressions [8]

$$
\zeta_{H}(0)=\frac{v_{t h}(0)}{\lambda_{0}}\left(1-\alpha^{2}\right) \sqrt{\frac{\pi}{2}}\left[1+\frac{3}{16} a_{2}(\alpha)\right]
$$

where the coefficient $a_{2}(\alpha)$ is

$$
a_{2}(\alpha)=\frac{16(1-\alpha)\left(1-2 \alpha^{2}\right)}{57-25 \alpha+30 \alpha^{2}-30 \alpha^{3}} .
$$

We have verified that the prediction in Eq. (2.12) is good also for the inhomogeneous DSMC and for MD.

\subsection{Simulation schemes}

The system described above is naturally simulated by means of an event-driven molecular dynamics algorithm (MD) where randomness is limited to the initial conditions (extracted from the 
canonical ensemble at temperature $T_{g}(0)$ ) and the evolution is deterministic: collisions occur at contact and rule (2.1) is instantaneously applied. A different scheme, the DSMC, involves an approximation of the above dynamics, where external noise is introduced when computing the effect of collisions. The algorithm involves the simulation of $\tilde{N}=f N$ fictive particles with $f>1$. This is a trick often used in DSMC: for molecular gases - where $N \sim 10^{20}$ - it is customary to use $f \ll 1$ in order to have a manageable number of fictive particles. In granular gases, where the number of real particles is small, one uses $f>1$ in order to have enough particles in a cell to guarantee Molecular Chaos. This is our case. For the sake of completeness, here we sketch the basic steps of the algorithm. As initial condition we have chosen a uniformly random spatial configuration of the particles and a Gaussian distribution of their velocities, as in MD. After initialization, the dynamics consists of a cycle with the following two steps:

- Streaming step (evolution of positions and velocities ignoring possible collisions)

- Collision step

The streaming step consists in computing, for every particle $i$, the time-discretized version (with a fixed $\Delta t$, small enough) of the evolution equations

$$
\begin{aligned}
\dot{\mathbf{r}}_{i} & =\tilde{\mathbf{v}}_{i} \\
\dot{\tilde{\mathbf{v}}}_{i} & =\omega_{0} \tilde{\mathbf{v}}_{i}
\end{aligned}
$$

For the purpose of the collision step, the system is partitioned in $m_{c}$ equal non-overlapping cells of size $l_{c}$ (an optimal value $l_{c} \sim 15 \sigma$ has been individuated, as discussed below). The average number of fictive particles in the cell $i$ is then $\tilde{N}_{c}=\tilde{N} / m_{c}$. In each cell $k$ the local average collision frequency - per particle - $\omega_{k}=\sigma n_{k} \sqrt{4 \pi T_{k} / m}$ is calculated, based on the real density $n_{k}=\tilde{N}_{k} /\left(f l_{c}^{2}\right)$ - where $\tilde{N}_{k}$ is the number of fictive particles in the cell - and on an estimate of the local temperature $T_{k}$ (i.e. the variance of the particle velocity distribution in the cell). Then a number of collisions $\tilde{N}_{k} \omega_{k} \Delta t$ is performed: this is obtained choosing, at random, pairs of particles $i$ and $j$, and unit vector (uniformly distributed angle) $\hat{\sigma}$, accepting them with a probability proportional to $\left(\mathbf{v}_{\mathbf{i}}-\mathbf{v}_{\mathbf{j}}\right) \cdot \hat{\sigma}$. The accepted pair is updated with equations (2.1). It should be noticed the deep difference in the fine collision mechanism between MD and DSMC: in the latter one draws randomly the unit vector $\hat{\sigma}$ and the real coordinates of the particles are ignored. Particles not at contact may always collide, if in the same cell. Most importantly, if the number of fictive particles $\tilde{N}_{c}$ in the cell is small (i.e. of order 1), it is possible to observe fake recollisions, i.e. particles which after a collision immediately re-collide. This is a spurious effect which certainly is not present in MD. The algorithm described here is the classical DSMC algorithm as proposed by Bird [2] (variant exist which include fluctuations of the number of collisions with the introduction of an internal clock for each cell). Incidentally we notice that, in this particular case, the number $f$, when integer and larger than 1, can be thought as a number of "copies" of the system. In fact, if $\Delta t$ is small enough to guarantee that $N_{k} \omega_{k} \Delta t \ll N_{k}$ for any cell $k$ (which is always true in our simulations), then one can always virtually separate the $\tilde{N}_{k}$ fictive particles of the cell into $f$ groups of - averagely - $N_{k}$ particles, such that collisions occur only between particles of the same 
group. Given that colliding pairs are chosen at random among the $\tilde{N}_{k}$ particles, at each step this virtual separation into $f$ groups is done in a new way, which is equivalent to say that - at each step - some of the particles move from one group to the other, remaining of course in the same cell. This is just an alternative representation of the algorithm, which underlines the strong randomizing mechanism - intrinsic of DSMC and not present in MD. Note that the last discussion does not imply that we have followed a different implementation of the DSMC algorithm: our recipe is exactly the one described above (streaming step and collision step).

\section{Spatial resolution and molecular chaos}

The basic definition of Molecular Chaos enters in the derivation of the Boltzmann equation. In the dilute limit one expects that the joint probability $P_{2}\left(\mathbf{v}_{1}, \mathbf{v}_{2}\right)$ of finding two colliding particles with velocities $\mathbf{v}_{1}$ and $\mathbf{v}_{2}$ factorizes into the product of two single particles probability $P_{1}\left(\mathbf{v}_{1}\right) P_{1}\left(\mathbf{v}_{2}\right)$. It is quite difficult to obtain a direct check of this assumption [22]. A better procedure is to verify the consequences of it in terms of simpler observables (this of course is a necessary but not sufficient check of Molecular Chaos). The DSMC algorithm, is a good tool to numerically solve Boltzmann equations, thanks to the stochastic computation of collisions: pairs of particles and their orientation vector $\hat{\sigma}$ are chosen randomly, with probabilities dictated by the Boltzmann collision integral. In homogeneous configurations, a pair of particles is chosen among all particles of the system, disregarding spatial coordinates: the large number of particles guarantees Molecular Chaos. Here, to avoid shear mode instabilities, we keep the total size of the system under the critical size $L_{c}$, expecting to observe a homogeneous regime. Nevertheless, the quantity under scrutiny, $U_{\perp}(t)$, requires that collisions are treated with a good spatial resolution, i.e. colliding particles must be close to each other to give the correct contribution to the variation of $U_{\perp}(t)$. For this reason, a inhomogeneous DSMC algorithm is necessary. The system is partitioned in $m_{c}$ non-overlapping cells of size $l_{c}$. The resolution for the measure of $U_{\perp}(t)$ is improved increasing $m_{c}$; at the same time, when $m_{c}$ increases, the average number of particles in each cell $N_{c}=N / m_{c}$ decreases, threatening the Molecular Chaos assumption. In principle the perfect resolution would be achieved with $l_{c} \sim \sigma$, but this would result in a number of particles per cell $N_{c} \sim n \sigma^{2}$ which, for diluteness, is required to be much smaller than 1. We will see in the following (see Fig. 2) that taking $l_{c} \sim 15 \sigma$ is sufficient to restore a good resolution for measuring $U_{\perp}(t)$. Such a choice, however, at the chosen packing fraction $n \sigma^{2}=10^{-2}$ gives $N_{c} \sim 2.5$, which is so low that the Molecular Chaos could be invalidated. A first check is to study the sensitivity of the rescaling procedure (2.7) (tested in [8] in the homogeneous DSMC simulations and in [7]) to the parameter $m_{c}$ (or equivalently $N_{c}$ ). Having this aim, we have characterized the entire system analyzing its granular temperature $T_{g}$ as function of the rescaled time $\tau$ for different choices of the cell number $m_{c}$ at $\alpha$ and $N$ fixed (see Fig. 1), comparing it with MD results. The time-rescaling is applied with a choice of $\omega_{0}$ such that, in the steady state it is expected $T_{g}=T_{0}$. From the simulation data it results that a steady state is always reached, i.e. the granular temperature is constant in all cases. A homogeneous DSMC (i.e. $m_{c}=1$ ) gives an agreement with the expected value which is better, of a few percentages, with respect to MD. When $m_{c}$ is increased, however, the DSMC gives very bad results. This is due to the 
small value of $N_{c}$, which results in strong finite size effects, for instance fake re-collisions which invalidate the Molecular Chaos hypothesis. The way of solving this problem is to simulate a larger

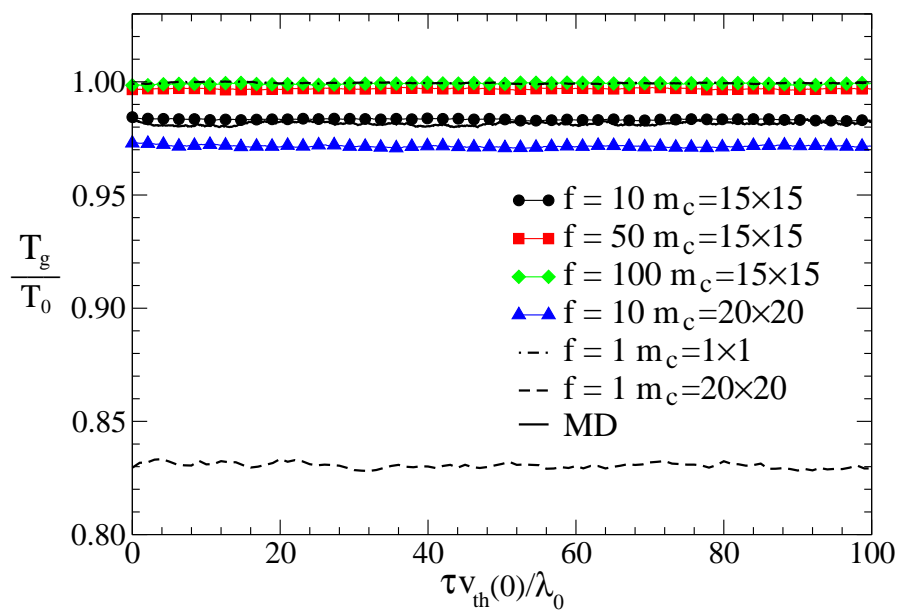

Figure 1: The rescaled granular temperature $T_{g}$ as function of the rescaled time, in DSMC simulations with different $f$ factors and cell numbers $m_{c}$, compared with MD simulations, in the case of $N=1000, \alpha=0.8$ and $n \sigma^{2}=0.01$.

number of particles $\tilde{N}=f N$ with $f>1$, keeping the collision statistics per particle as that of a $N$-particles system (see previous section for a precise description). This is equivalent to simulate $f$ copies of the original $N$-particles system and let particles of different copies swap at each time step. The effect of $f>1$ is immediately seen in Fig. 1: the steady state granular temperature perfectly agrees with that measured in MD, as soon as $f \sim 10$, even if $m_{c}=225$. Much larger values of $f$ appear to slightly improve the value of $T_{g}$. On the other side a too much large value of $f$ can be very expensive in terms of cpu-time. A crucial quantity, here, is the number of virtual particles per cell $\tilde{N}_{c}=f N_{c}=f N / m_{c}$. If $m_{c}$ is increased (for instance in Fig. 1 it is changed from 225 to 400), $f$ should be increased. Apparently it is sufficient to have $\tilde{N}_{c} \sim 25$ to have a good agreement, for $T_{g}$, between DSMC and MD. As discussed above, the necessity of a large number of cells comes from the sensitivity of $U_{\perp}(t)$ to the spatial localization of collisions. This is shown in Fig. 2, where the decay of $C_{\perp}(\tau)$ is displayed: the homogeneous DSMC $\left(m_{c}=1\right)$ shows an exponential decay with a $\tau_{0}$ completely different from the one observed in the MD simulations. This behavior is insensitive to changes of $f$ from 1 to 10 . In order to obtain the correct decay, it is necessary to use the inhomogeneous DSMC approach with $m_{c} \gg 1$. A partition of $m_{c}=20 \times 20$ for $N=1000$, in fact, reveals an exponential decay very close to the MD results: also in this case, a change of $f$ from 10 to 100 is unimportant. When $N$ is increased at fixed density (and mean free path), the size of cells must be kept constant, which implies $m_{c} \propto N$. If $f$ is also kept constant, $\tilde{N}_{c}$ is automatically preserved and we expect to have a good comparison with MD simulations in terms of Molecular Chaos, as well as decay of $C_{\perp}(\tau)$. This situation is fairly verified in Fig. 3, where the decay of $C_{\perp}(\tau)$ is shown to be always close to the MD results. This approach is valid in general and, with proper values of $f$ and $m_{c}$, we can compare, for different values of $\alpha$ and 


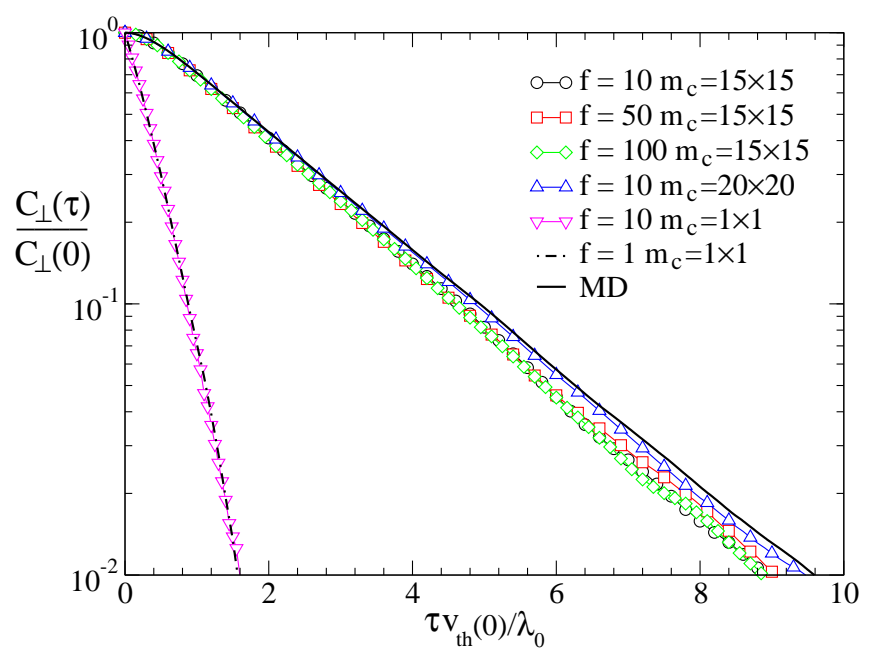

Figure 2: The rescaled correlation function $C_{\perp}(\tau)$ (see Eq. 2.9) as function of the rescaled time, obtained from DSMC simulations with different $f$ factors and cell numbers $m_{c}$, and from MD simulations, in the case of $N=1000, \alpha=0.8$ and $n \sigma^{2}=0.01$.

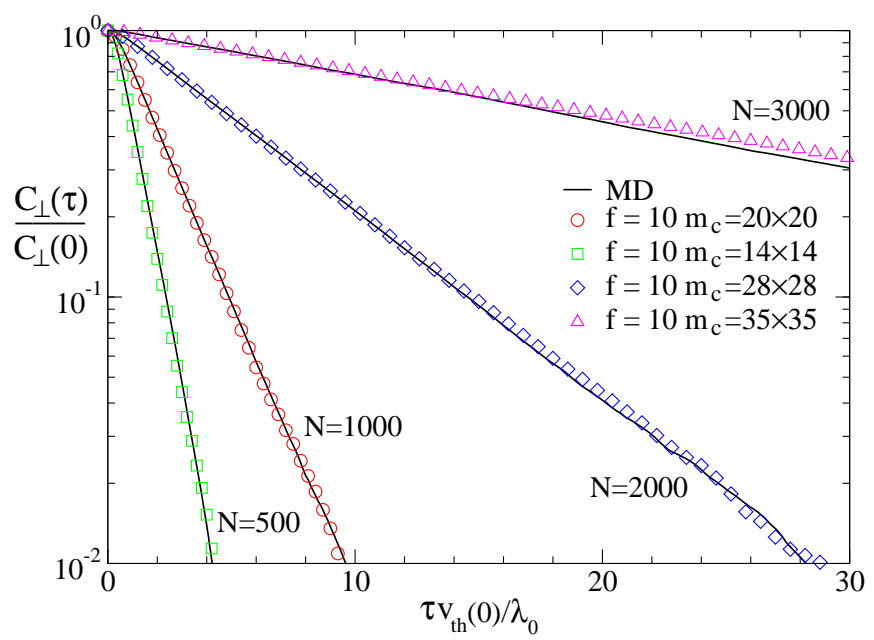

Figure 3: The rescaled correlation function $C_{\perp}(\tau)$ as function of the rescaled time, from DSMC simulations with different total particle numbers $N$ and same average number of particles per cell $\tilde{N}_{c}$. Data from MD simulations are shown for comparison. The parameters are the same as in Fig. 1. 
$N$, the properties of the fluctuations of $W_{\perp}$ between DSMC and MD simulations. Guided by this preliminary analysis, in the following section we have studied the role of the parameters $f$ and $\tilde{N}_{c}$ in the DSMC scheme.

\section{Hydrodynamic noise from numerical simulations}

The aim of this section is to measure $\tilde{\nu}$ and $c$ by means of Equations (2.10) and (2.11) in DSMC and MD simulations, obtaining a comparison. We start using optimal parameters found in the previous section, to guarantee the validity of the HCS assumptions to measure of $W_{\perp}(\tau)$ also in the DSMC. Eq. (2.9) is expected to be valid in the large $N$ limit: for this reason we have
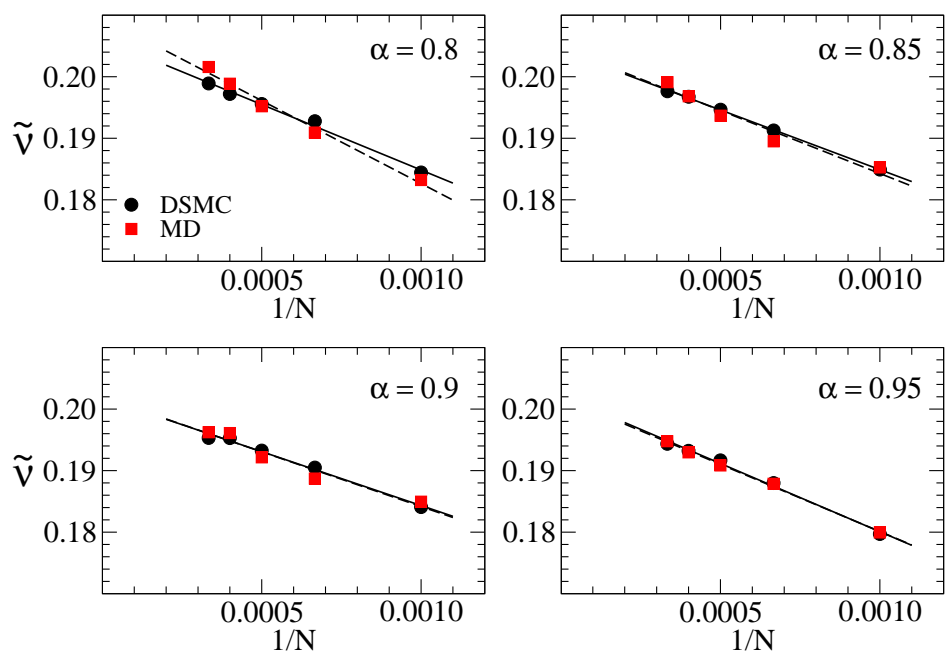

Figure 4: The dimensionless kinematic viscosity $\tilde{\nu}$, obtained from Eq. (2.10) and from the exponential decay of the correlation function in Eq. (2.9), as function of the inverse of the number of particles $N$ for different values of $\alpha$. The circles and squares correspond to data obtained from DSMC an MD simulations, respectively. The solid and dashed lines are the best linear fit. The density is the same as in Fig. $1, f=10$ and $\tilde{N}_{c}=25$.

performed simulations for different values of $N(1000,1500,2000,2500$ and 3000), fixing the particle density $n$ of the system and the parameters $f$ and $\tilde{N}_{c}$, and measuring the time decay $\tau_{0}$. The data obtained using (2.10) and (2.12) in DSMC and MD, are shown in Fig. 4. The dimensionless viscosity $\tilde{\nu}$ appears to be a linear function of $N^{-1}$, therefore a best linear fit allows to extrapolate the asymptotic value for $N \rightarrow \infty$. The agreement between MD and DSMC data is good in all cases, even at not too high values of $N$. Our second question is if the inhomogeneous DSMC can reproduce the MD results for the noise term in the Langevin equation (2.3) or (2.8), in particular the quantity $\tilde{\nu}^{\prime} \equiv c \tilde{\nu}$ that is the viscosity term appearing in the noise. Analogously to Fig. 4, we have analyzed its dependence versus $N^{-1}$. Also in this case we have found a linear law, shown in Fig. 5: a small discrepancy is observed, for this quantity, between DSMC and MD data, when 
$N$ is increased. The disagreement results more evident if the collisions are more inelastic. Our interpretation of this small difference is that the DSMC dynamics at small time-scales is slightly different from MD: indeed, this disagreement is less evident at large time-scales, for instance in the measure of $\tilde{\nu}$. If we consider also that the DSMC and MD results about the correlation of the energy fluctuations are very similar [37], we tend to conclude that this small discrepancy could be due to the use of small cells (large $m_{c}$ or equivalently small $\tilde{N}_{c}$ ), which is necessary in the measures of $C_{\perp}(0)$. A more general discussion of the cause of this discrepancy is given at the end of this section.
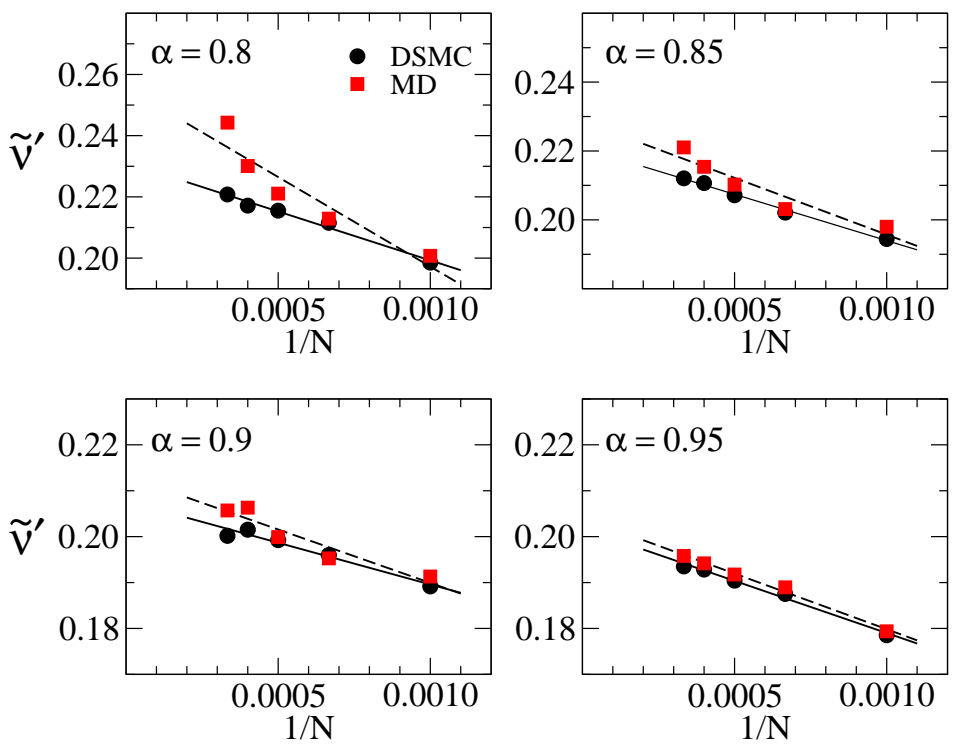

Figure 5: The dimensionless viscosity $\tilde{\nu}^{\prime}=c \tilde{\nu}$ obtained from Eq. (2.11) and (2.10) (see text), as function of the inverse of the number of particles $N$ for the following values of $\alpha: 0.8,0.85,0.9$ and 0.95. The circles and squares correspond to data obtained from DSMC an MD simulations, respectively. The solid and dashed lines are the best linear fit. The density is the same as in Fig. 1, $f=10$ and $\tilde{N}_{c}=25$.

\subsection{Violation of FDR}

Notwithstanding this slight discrepancy, the value of $c=\tilde{\nu}^{\prime} / \tilde{\nu}$ results significantly larger than 1 in both DSMC and MD. The inhomogeneous DSMC is then able to reproduce the breakdown of the FDR as already found with the MD approach [3]. This is evident from Fig. 6 where the ratio $c$ is shown (focus for the moment on black full circles, DSMC, and red full squares, MD): it clearly displays strong deviations from 1 (violations of FDR), which reach $12 \%$ at $\alpha=0.8$ also in DSMC. The dot-dashed curve represents the result for $c$ from the theoretical analysis of [5] with the additional assumption of white noise. We note a close comparison with the data from DSMC, suggesting that the effect of this protocol is an effective time-decorrelation of noise. Such 
a conclusion should be taken with care, in view of other simulations, performed with different choices of $f$ and $m_{c}$, discussed in the next section. The presence of time-correlation in the noise,

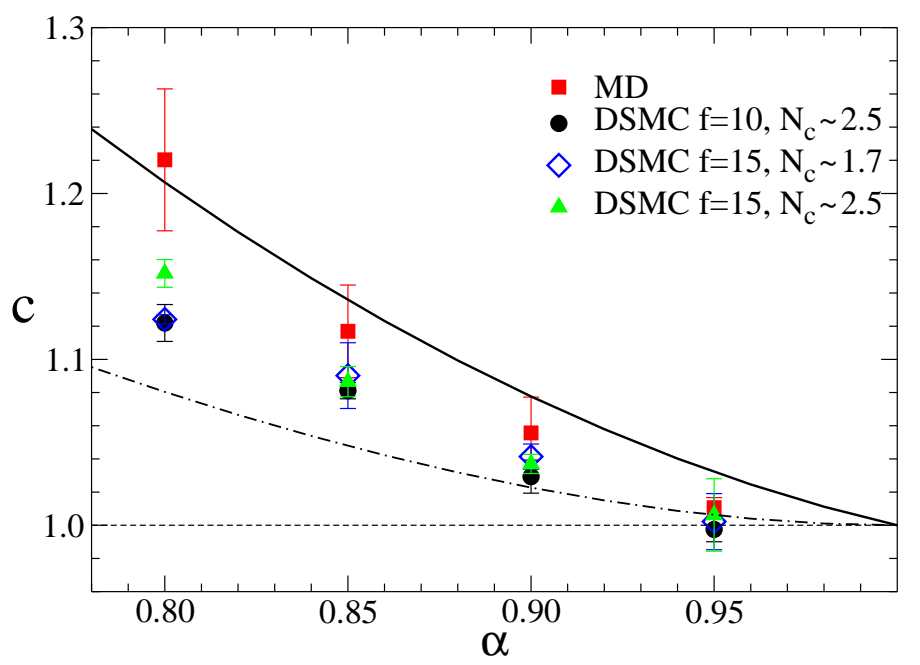

Figure 6: The ratio $c=\tilde{\nu}^{\prime} / \tilde{\nu}$ as function of the coefficient of restitution $\alpha$ for $N \rightarrow \infty$. The squares correspond to MD data while the DSMC data are shown for three different cases: a) $f=10, N_{c}=2.5$ and $\tilde{N}_{c}=25$ (circles); b) $f=15, N_{c}=1.7$ and $\tilde{N}_{c}=25$ (diamonds); c) $f=15, N_{c}=2.5$ and $\tilde{N}_{c}=37.5$ (triangles). The solid line is the theoretical prediction while the dot-dashed line is the theoretical prediction with the assumption of white noise [5]. The value of reference $c=1$ (dashed line) is obtained if FDR are satisfied.

even in DSMC, is in agreement with the results for $C_{\perp}(\tau)$ shown in Fig. 2 and 3, which are not straight exponentials, but some bending can be observed at small $\tau$. Apart from these small discrepancies, the main fact is that the inhomogeneous DSMC is able to reproduce the violation of FDR, which was not obvious. In fact, it is reasonable and well verified that average values (e.g. transport coefficients) obtained in DSMC agree with dilute MD simulations; anyway it is less trivial to observe good agreement for fluctuations, which - in non-equilibrium situations - can be much more sensitive to the detailed mechanisms of the dynamics.

\subsection{Improvement of Molecular Chaos}

We have also performed other DSMC simulations with different choices of the parameters $m_{c}$ and $f$ : we remind that an increase of $m_{c}$ improves the spatial resolution of measurements but negatively affects molecular chaos, while an increase of $f$ usually improves Molecular Chaos by reducing the number of fake recollisions. Indeed our results are consistent with this interpretation and with the underlying idea that DSMC, in the good limit, reproduces MD fluctuations at all timescales. These results are presented in Fig. 6. First we have tried to increase the spatial resolution, decreasing $N_{c}=N / m_{c}$, but keeping fixed the number of fictive particles per cell $\tilde{N}_{c}=f N_{c}$, by increasing simultaneously $f$, see empty blue diamonds in the figure. Remarkably this choice of 
parameters fairly agrees with the results discussed in the previous section: discrepancies from MD and kinetic theory are still visible at small inelasticity. Second, we have increased $\tilde{N}_{c}$ keeping fixed $N / m_{c}$, which is obtained by increasing only $f$ (see green full triangles). In this case we expect to reduce the (already small) incidence of fake recollisions. This procedure increases the agreement between DSMC and MD for two choices of inelasticity ( $\alpha=0.8$ and $\alpha=0.95$ ), and leaves mainly unaltered the other cases. The strongest indication concerns the most inelastic case, where the gap between MD and DSMC was larger and where the increase of $f$ gives a neat improvement. These results suggest that the critical quantity to be adjusted in DSMC with the aim of reproducing hydrodynamic fluctuations at small and large time-scales (amplitude and asymptotic decay) is $\tilde{N}_{c}$, controlled by the parameter $f$.

\section{Conclusions}

In this work we have compared MD and DSMC results for some main properties of the hydrodynamic noise, focusing on the transverse velocity field at its largest (minimum wave number) mode. To obtain a proper comparison, we have revised the basic ingredients - in a Direct Simulation Monte Carlo algorithm - to correctly measure the time-decay of the autocorrelation function of fluctuations of a hydrodynamic field, keeping valid the assumption of Molecular Chaos [11]: the volume must be divided in small cells for the purpose of good spatial resolution in computation of collisions, even if the system is assumed to be spatially homogeneous. The good resolution to appreciate the correct time-decay of $C_{\perp}(\tau)$ is achieved only when $N_{c} \sim 2$ which is too small to avoid fake recollisions: for this reason, a number (order $\sim 10$ ) of "virtual" copies of the system is necessary to restore Molecular Chaos. This procedure is similar to that adopted in [6]. Notice that the study of the transverse velocity field could also be done by dividing the system in slides, as in $[10,17]$. Such a procedure can be used to analyze $U_{\perp}(\tau)$, while we have no knowledge of its adaptation to other space-dependent fields. The reader should also keep into account that when DSMC is used to study the homogeneous cooling state, i.e. when the system size is below the critical one for instability, $L<L_{c}$ - one usually does not divide the system in cells, and still obtains excellent agreement for one-point observables. Previous study of cooling granular gases with inhomogeneous DSMC [7, 6] where in fact proposed to analyze the departure from the HCS. Here we do not investigate states different from the HCS, nevertheless fine spatial resolution (inhomogeneous DSMC) is still necessary. The general message coming out from this numerical comparison is that observables such as the viscosity, which dictates the large time decay of the transverse velocity autocorrelation, are well reproduced both in MD and DSMC, caring only about the spatial resolution in the latter scheme. The amplitude of fluctuations, on the other side, which is related to two-particles statistics, shows discrepancies with respect to MD results, which become more evident as $\alpha$ is reduced and $N$ increases. These discrepancies depend on the choice of the DSMC parameter $\tilde{N}_{c}$ which measures the average number of fictive particles in a DSMC cell: an increase of this number, keeping constant the cell size, reduces the probability of recollisions and improves the agreement. In DSMC simulations, deviations from a perfect exponential decay of $C_{\perp}(\tau)$ are also visible, at small times, qualitatively similar to those observed and predicted in [5]: 
again the increase of $\tilde{N}_{c}$ seems to improve this fine feature. Our interpretation of these results is that the hydrodynamic noise in DSMC does not reproduce exactly the kinetic theory predictions, which are instead well fulfilled by MD simulations, but neither reproduces exactly the predictions of the theory with white noise (dot-dashed curve in Figure. 6). The noise amplitude indeed falls in between the two predictions and depends on some DSMC parameters, such as $f$ or $\tilde{N}_{c}$ which are usually considered only numerical parameters. It can be useful to resume how the main DSMC parameters influence the different observables, to get an idea of the required power of resolution necessary to get finer and finer details:

- homogeneous DSMC ( $\left.f=1, m_{c}=1\right)$ : it well reproduces the average kinetic energy predicted by HCS kinetic theory; it completely fails to reproduce the decay of velocity modes autocorrelations;

- inhomogeneous DSMC with no fictive particles $(f=1)$ and large number of cells $\left(m_{c} \sim\right.$ $15 \times 15$ ): it fails in comparing with the average kinetic energy predicted by HCS (inaccurate Molecular Chaos due to too many fake recollisions);

- inhomogeneous DSMC with fictive particles $f \sim 10$ and large number of cells $\left(m_{c} \sim\right.$ $15 \times 15$ ), corresponding to a number of fictive particles per cell $\tilde{N}_{c} \sim 25$ : it well reproduces the autocorrelation large-times decay; it shows discrepancies in the small-times features (amplitude and colour) of the noise;

- increase of $\tilde{N}_{c}$ by keeping constant $m_{c}$ (i.e. increase of $f$ ): improvement in small-times features of the hydrodynamic noise (amplitude and colour).

Hydrodynamic noise is always important in granular systems, which - in terms of number of elementary constituents - are much smaller than molecular fluids $\left(10^{4}\right.$ instead of $10^{20}$ particles $)$. Future research is needed to understand the properties of granular hydrodynamic noise in steady state models: even if we have shown that these properties do not depend on the detailed collisional mechanism, it seems that they depend on the choice of energy driving protocol [27].

\section{Acknowledgments}

The work of the authors is supported by the "Granular-Chaos" project, funded by the Italian MIUR under the FIRB-IDEAS grant number RBID08Z9JE.

\section{References}

[1] A. Barrat, V. Loreto, A. Puglisi. Temperature probes in binary granular gases. Physica A, 334 (2004), No. 3-4, 513-523.

[2] G. A. Bird. Molecular Gas Dynamics and the Direct Simulation of Gas Flows. Clarendon, Oxford, 1994. 
[3] J. J. Brey, M. I. Garcia de Soria, P. Maynar. Breakdown of the fluctuation-dissipation relations in granular gases. Europhys. Lett., 84 (2008), No. 2, 24002.

[4] J. J. Brey, J. W. Dufty, C. S. Kim, A. Santos. Hydrodynamics for granular flow at low density. Phys. Rev. E, 58 (1998), No. 4, 4638-4653.

[5] J. J. Brey, P. Maynar, M. I. Garcia de Soria. Fluctuating hydrodynamics for dilute granular gases. Phys. Rev. E, 79 (2009), No. 5, 051305.

[6] J. J. Brey, M. J. Ruiz-Montero. Validity of the boltzmann equation to describe low-density granular systems. Phys. Rev. E, 69 (2004), No. 1, 011305.

[7] J. J. Brey, M. J. Ruiz-Montero, F. Moreno. Instability and spatial correlations in a dilute granular gas. Phys. Fluids, 10 (1008), No. 11, 2976-2982.

[8] J. J. Brey, M. J. Ruiz-Montero, F. Moreno. Steady-state representation of the homogeneous cooling state of a granular gas. Phys. Rev. E, 69 (2004), No. , 051303-.

[9] J.J. Brey, M.I.G. de Soria, P. Maynar, M.J. Ruiz-Montero. Energy fluctuations in the homogeneous cooling state of granular gases. Phys. Rev. E, 70 (2004), No. 1, 011302.

[10] J.J. Brey, M.J. Ruiz-Montero. Average energy and fluctuations of a granular gas at the threshold of the clustering instability. Granular Matter, 10 (2007), No. 1, 53-59.

[11] G. Costantini, A. Puglisi. Fluctuating hydrodynamics for dilute granular gases: a Monte Carlo study. Phys. Rev. E, 82 (2010), No. 1, 011305.

[12] G. Costantini, A. Puglisi, U. Marini Bettolo Marconi. Granular Brownian ratchet model. Phys. Rev. E, 75 (2007), No. 6, 061124-.

[13] G. Costantini, A. Puglisi, U. Marini Bettolo Marconi. Velocity fluctuations in a one dimensional inelastic Maxwell model. J. Stat. Mech., (2008), P08031.

[14] J. W. Dufty, J. J. Brey. Green-Kubo expressions for a granular gas. J. Stat. Phys., 109 (2002), No. 3-4, 433-448.

[15] J. Eggers. Sand as Maxwell's demon. Phys. Rev. Lett., 83 (1999), No. 25, 5322-5325.

[16] K. Feitosa, N. Menon. Breakdown of energy equipartition in a 2d binary vibrated granular gas. Phys. Rev. Lett., 88 (2002), No. 19, 198301.

[17] A. L. Garcia, M. Malek Mansour, G. C. Lie, M. Mareschal, E. Clementi. Hydrodynamic fluctuations in a dilute gas under shear. Phys. Rev. A, 36 (1987), No. 9, 4348-4355.

[18] I. Goldhirsch. Scales and kinetics of granular flows. Chaos, 9 (1999), No. 3, 659-672.

[19] I. Goldhirsch, G. Zanetti. Clustering instability in dissipative gases. Phys. Rev. Lett., 70 (1993), No. 11, 1619-1622. 
[20] R. Kubo, M. Toda, N. Hashitsume. Statistical physics II: Nonequilibrium stastical mechanics. Springer, Berlin, 1991.

[21] L. D. Landau, E. M. Lifchitz. Physique Statistique. Éditions MIR, Moscow, 1967.

[22] J. F. Lutsko. Molecular chaos, pair correlations, and shear-induced ordering of hard spheres. Phys. Rev. Lett., 77 (1996), No. 11, 2225-2228.

[23] J. F. Lutsko. A model for the atomic-scale structure of the homogeneous cooling state of granular fluids. Phys. Rev. E, 63 (2001), No. 6, 061211.

[24] M. Mansour Malek, A. L. Garcia, G. C. Lie, E. Clementi. Fluctuating hydrodynamics in a dilute gas. Phys. Rev. Lett., 58 (1987), No. 9, 874-877.

[25] U. Marini Bettolo Marconi, A. Puglisi. Mean-field model of free-cooling inelastic mixtures. Phys. Rev. E, 65 (2002), No. 5, 051305.

[26] U. Marini Bettolo Marconi, A. Puglisi, L. Rondoni, A. Vulpiani. Fluctuation-dissipation: Response theory in statistical physics. Phys. Rep., 461 (2008), No. 4-6, 111-195.

[27] P. Maynar, M. I. G. de Soria, E. Trizac. Fluctuating hydrodynamics for driven granular gases. Eur. Phys. J. Special Topics, 170 (2009), No. 1, 123-139.

[28] R. Pagnani, U. Marini Bettolo Marconi, A. Puglisi. Driven low density granular mixtures. Phys. Rev. E, 66 (2002), No. 5, 051304.

[29] T. Pöschel, N. Brilliantov, editors. Granular Gas Dynamics. Lecture Notes in Physics 624. Springer, Berlin, 2003.

[30] T. Pöschel, S. Luding, editors. Granular Gases. Lecture Notes in Physics 564. Springer, Berlin, 2001.

[31] A. Puglisi, A. Baldassarri, V. Loreto. Fluctuation-dissipation relations in driven granular gases. Physical Review E, 66 (2002), No. 6, 061305.

[32] A. Puglisi, A. Baldassarri, A. Vulpiani. Violations of the Einstein relation in granular fluids: the role of correlations. J. Stat. Mech., (2007), P08016.

[33] A. Sarracino, D. Villamaina, G. Costantini, A. Puglisi. Granular brownian motion. J. Stat. Mech., (2010) P04013.

[34] A. Sarracino, D. Villamaina, G. Gradenigo, A. Puglisi. Irreversible dynamics of a massive intruder in dense granular fluids. Europhys. Lett., 92 (2010), No. 3, 34001.

[35] T. C. P. van Noije, M. H. Ernst, R. Brito, J. A. G. Orza. Mesoscopic theory of granular fluids. Phys. Rev. Lett., 79 (1007), No. 3, 411-414. 
[36] D. Villamaina, A. Puglisi, A. Vulpiani. The fluctuation-dissipation relation in sub-diffusive systems: the case of granular single-file diffusion. J. Stat. Mech., (2008), L10001.

[37] P. Visco, A. Puglisi, A. Barrat, F. van Wijland, E. Trizac. Energy fluctuations in vibrated and driven granular gases. Eur. Phys. J. B, 51 (2006), No. 3, 377-387. 